EPJ Web of Conferences 73, 01002 (2014)

DOI: $10.1051 /$ epjconf/20147301002

(C) Owned by the authors, published by EDP Sciences, 2014

\title{
Latest results from meson photoproduction at ELSA and MAMI
}

\author{
B. Krusche ${ }^{\text {a }}$ for the CBELSA/TAPS- and A2-collaborations \\ Department of Physics, University of Basel, 4056 Basel, Switzerland
}

\begin{abstract}
Photoproduction of mesons plays a key role for the investigation of the excitation spectrum of the nucleon and thus for our understanding of the strong interaction in the nonperturbative regime. In this contribution we discuss recent results from the experiments at the tagged photon beams of the electron accelerators ELSA in Bonn and MAMI in Mainz. They include the measurement of cross sections and (double) polarization observables for single meson production and production of meson pairs off free protons as well as of quasifree nucleons bound in light nuclei (in particular the deuteron).
\end{abstract}

\section{Introduction}

The excitation spectrum of a bound system of particles is expected to reflect the properties of the underlying interaction. Well-known examples are coming from atomic and nuclear physics. A similar approach for the relation between the excited states of nucleons and the fundamental properties of Quantum Chromodynamics (QCD) had so far much more limited success. Recent progress, however, is significant. Fully relativistic quark model approaches have been developed [2], and the application of the Dyson-Schwinger approach to QCD has led to promising results [3, 4]. The advances in lattice gauge calculations allowed first predictions of the excitation spectrum [5]. Interestingly, they "re-discovered" the $\mathrm{SU}(6) \otimes \mathrm{O}(3)$ excitation structure of the nucleon with a level counting consistent with the standard non-relativistic quark model.

Efforts on the experimental side aimed at a better data base for the excited nucleon states. A few years ago the listing of $N^{\star}$ (isospin $\left.I=1 / 2\right)$ and $\Delta^{\star}(I=3 / 2)$ resonances in the Review of Particle Physics (PDG) included only states that had been observed in inelastic pion scattering reactions. Only in the most recent update [6] states "established" by photon induced reactions were included. Possible bias for states with weak coupling to $N \pi$ are obvious. The experimental efforts during the last decade, which are still continuing, tried to remove such bias by a large-scale investigation of meson-photoproduction for different final states, including production of meson pairs. This program includes the measurement of single and double polarization observables.

The Bonn ELSA and Mainz MAMI experiments deliver linearly and circularly polarized photon beams and are equipped with polarized targets (longitudinally or transversely polarized protons or

\footnotetext{
${ }^{a}$ e-mail: Bernd.Krusche@unibas.ch
}

This is an Open Access article distributed under the terms of the Creative Commons Attribution License 4.0, which permits unrestricted use, distribution, and reproduction in any medium, provided the original work is properly cited. 

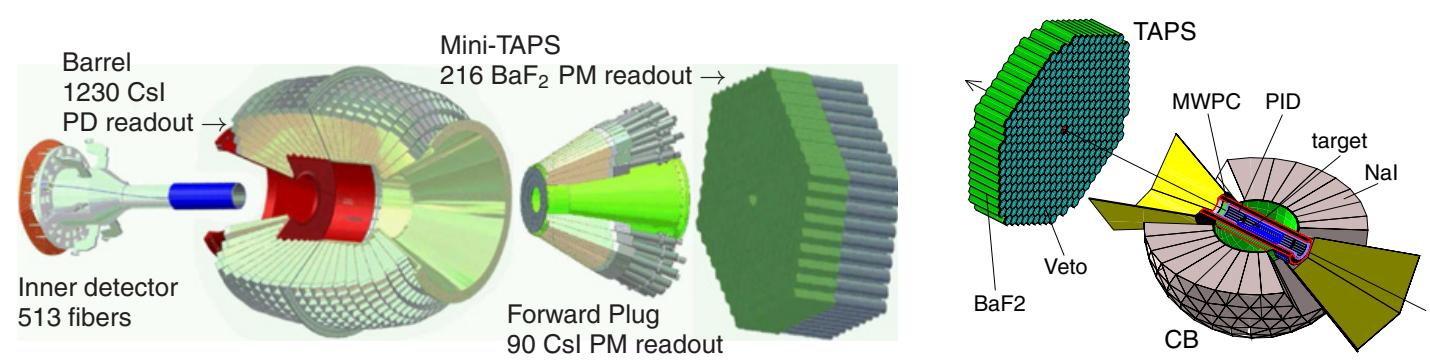

Figure 1. Setup of the Crystal Barrel/TAPS detector at ELSA (left hand side) and the Crystal Ball/TAPS experiment at MAMI.

deuterons). They are complementary to other tagged photon facilities like for example CLAS at Jlab, which is optimized for the detection of charged particles. The experiments discussed here are also sensitive to final states with two or more neutral mesons even when they are produced off the neutron.

\section{Experiments}

The two experiments are similar, one of the main differences lies in the accelerator specifications. ELSA [7] can deliver electron beams with energies up to $3.5 \mathrm{GeV}$ of relatively low intensities (order of $\approx 1 \mathrm{nA}$ ), while the cascaded race-track microtron [8] in Mainz (also used for electron scattering) reaches energies up to $1.5 \mathrm{GeV}$ with intensities up to $\approx 100 \mu \mathrm{A}$, far beyond what is needed ( $\approx$ few tens of $\mathrm{nA}$ ) for taggedphoton beams.

Tagged photon beams are produced by scattering the electrons in a radiator to produce bremsstrahlung, which is energy tagged by a momentum analysis of the scattered electrons in magnetic spectrometers. The tagging device at MAMI is described in detail in [9]. Both accelerators can deliver longitudinally polarized electron beams, which are used to produce circularly polarized photon beams. Linearly polarized photon beams are produced with coherent bremsstrahlung from a diamond lattice [10]. Polarized target nucleons are available from buthanol targets in frozen-spin mode [11].

The reaction products are detected with almost $4 \pi$ covering electromagnetic calorimeters, schematically shown in Fig. 1. Their large angle coverage and good detection properties for photons, neutrons, and charged particles is the basis of the versatile experimental programs. The largest part of the solid angle is covered by the more or less spherical calorimeter parts - the Crystal Barrel (CsI scintillators) [13] at ELSA and the Crystal Ball (NaI crystals) [12] at MAMI combined with forward scintillator walls of $\mathrm{BaF}_{2}$ crystals from the TAPS detector [14]. The setups are complemented by detectors for charged particle identification and tracking mounted around the targets and in front of the crystals from the TAPS detector.

\section{Experimental results}

\subsection{Photoproduction of $\pi^{0}$-mesons}

Historically, pion scattering or single pion production where the backbone for the study of nucleon resonances. In photoproduction the $N \pi^{0}$ channel is very interesting because it has much less smaller contributions from non-resonant backgrounds than reactions involving charged pions. Recent new data for several polarization observables for the $\gamma p \rightarrow p \pi^{0}$ reaction had significant impact. The previously available data base had been analyzed with different reaction models and partial wave analyses like e.g. MAID, SAID, and the Bonn-Gatchina model (BnGn) [16-18]. However, already the results for the first double polarization observable $(G)$ measured at ELSA [20] made a readjustment of the model fits 

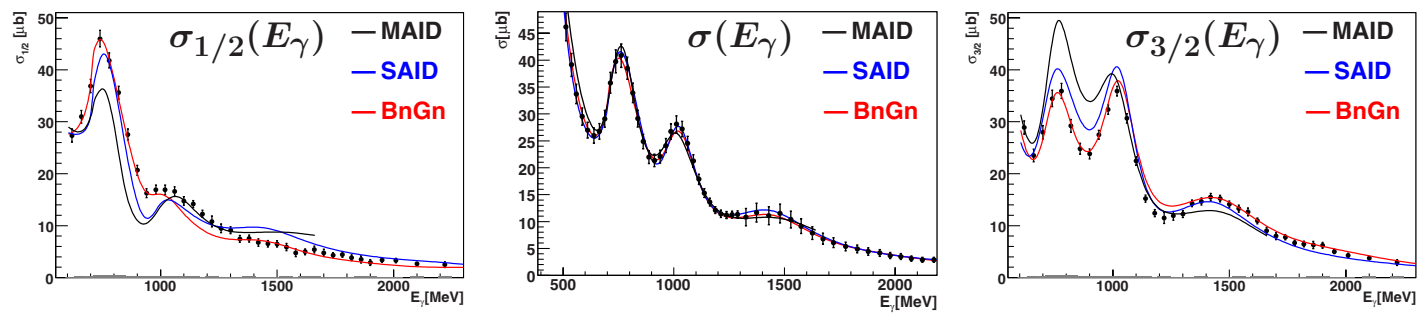

Figure 2. Split of the total cross section (center) of $\gamma p \rightarrow p \pi^{0}$ into the helicity-1/2 (left hand side) and helicity-3/2 (right hand side) components [15] (ELSA). Theory curves from MAID [17], SAID [16], and BnGn [18].

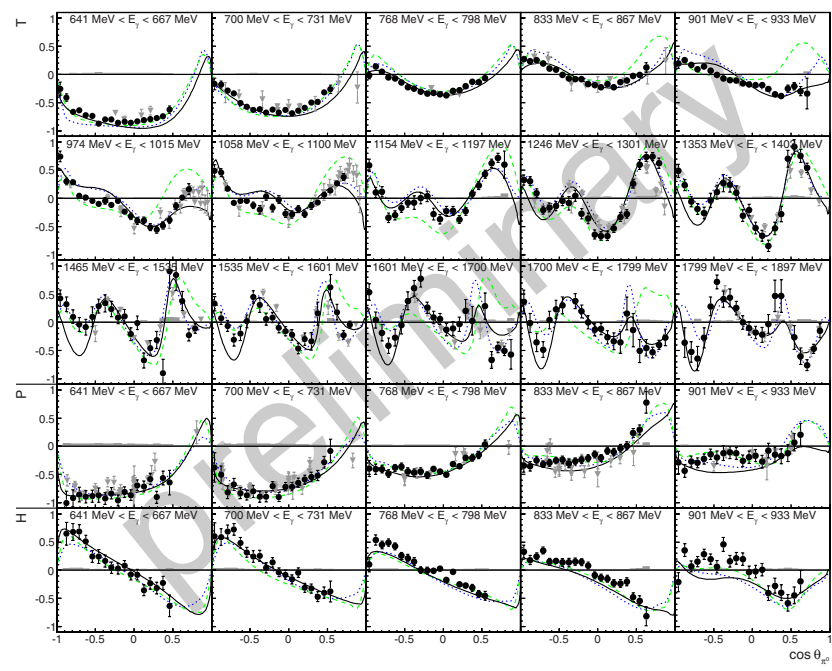

Figure 3. Examples for the polarization observables $T$ (target asymmetry, transversely polarized target), $P$ (recoil polarization) and $H$ both measured as double polarization observables with transversely polarized target and linearly polarized beam) (ELSA). Black dots: present results, grey triangles: previous results for $T$ and $P$ [21], curves: MAID (dashed) [17], SAID (dotted) [16], and BnGn (solid) [18].

necessary. The largest effects were found at quite low incident photon energies for the $E_{0+}$ and $E_{2-}$ partial waves involving excitation e.g. of the $S_{11}(1535)$ and $D_{13}(1520)$ nucleon resonances.

Here, we discuss another polarization degree of freedom, namely the helicity decomposition of the total cross section. This is measured with a circularly polarized photon beam on a longitudinally polarized target. Parallel alignment of photon and nucleon spin corresponds to a spin projection on the beam axis of $3 / 2$ (only possible for nucleon resonances with $J \geq 3 / 2$ ), while with antiparallel alignment also $J=1 / 2$ states may be excited. The unpolarized cross section and the $\sigma_{1 / 2}$ and $\sigma_{3 / 2}$ components measured at ELSA [15] are compared in Fig. 2 to model predictions. The models agree with the previously known unpolarized cross section, but the spilt into helicity components is quite different. It is obvious, that further measurements of polarization observables are necessary for a convergence of the different analyses approaches. As an example for the already achieved quality of such data we show in Fig. 3 some preliminary data measured at ELSA for different polarization observables.

A complete determination of the amplitudes involved in photoproduction of a pseudo-scalar meson requires also the measurement of observables involving the polarization state of the recoil nucleon. Here, recently progress has been made at the MAMI facility using a novel large-acceptance recoil nucleon polarimeter based on the scattering process off Carbon nuclei. As a first test the observable $C_{x}^{\star}$ involving circularly polarized photons and the measurement of the recoil proton polarization has been measured for the $\gamma p \rightarrow p \pi^{0}$ reaction [22].

Much less data is so far available for the photoproduction of pions off neutrons. The isospin decomposition of the cross section is so far entirely based on the measurement of the $\gamma n \rightarrow p \pi^{-}$reaction 

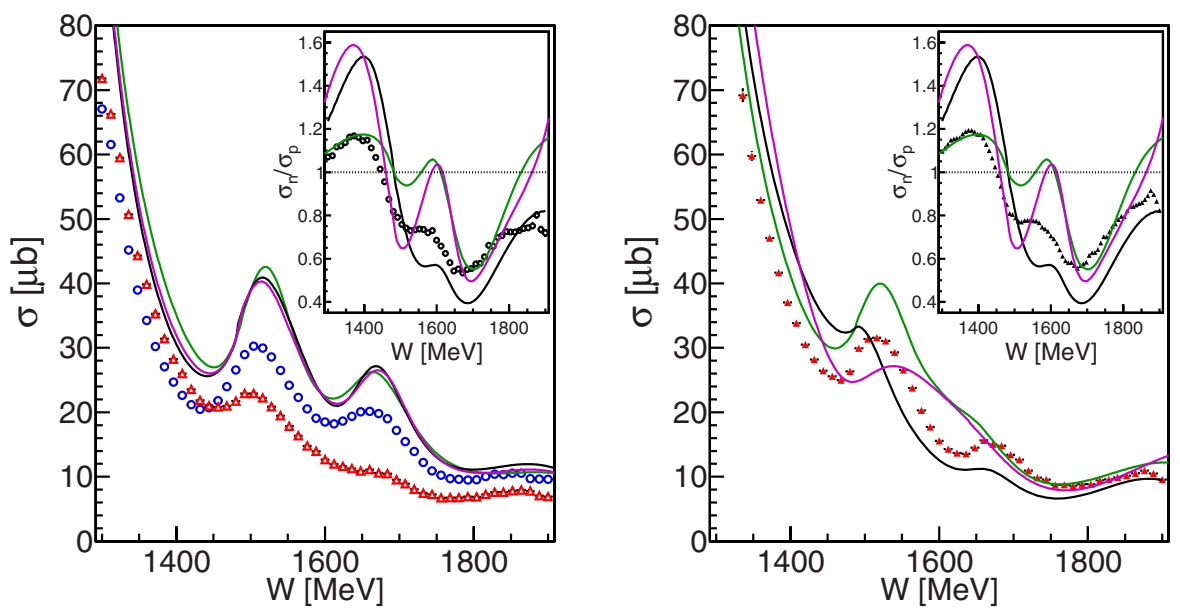

Figure 4. Total cross sections of $\gamma N \rightarrow N \pi^{o}$ for photoproduction of quasi-free nucleons bound in the deuteron (MAMI). Right hand side: quasi-free proton (blue circles) and quasi-free neutron (red triangles) compared to model predictions for free proton target (black: SAID [16], green MAID [17], magenta BnGn [18, 19]). Right hand side: FSI corrected (see text) results for neutron target compared to model predictions for free neutron. Inserts: neutron/proton ratios. All results preliminary.

off quasi-free neutrons [23, 24]. However, in this reaction channel contributions from non-resonant background terms are much stronger than for $\gamma n \rightarrow n \pi^{0}$. Preliminary results for a first measurement of the latter reaction in quasi-free kinematics using a deuteron target are available from MAMI. The final state invariant mass $W$ has been deduced from the completely determined reaction kinematics as discussed in [25], so that effects from nuclear Fermi motion were eliminated.

Total cross sections are compared in Fig. 4 to model predictions. The left hand side of the figure shows the quasi-free proton and neutron cross sections, which are quite different Also shown at the left hand side are the results for the MAID [17], SAID [16], and BnGn [18] analyses for the free proton. The three curves agree among each other (because all have been fitted to the same free-proton data) but not with the quasi-free proton data. This is evidence for final state interaction (FSI) effects, which previously were already discussed for the $\gamma d \rightarrow X \pi^{0}$ reaction in the $\Delta$ resonance range [26]. Model estimates for FSI at higher incident photon energies are not yet available for this channel. However, as a first approximation one can assume, that the effects are similar for bound protons and bound neutrons and correct the neutron data with $W$ - and angle-dependent factors derived from the comparison of free and quasi-free proton data. The result is compared to the model predictions at the right hand side of Fig. 4. The model results do not agree among each other and none of them is in close agreement with the data, indicating that the analysis of the $p \pi^{-}$final state is not sufficient to fix the isospin decomposition of the reaction.

The measurement of single and double polarization observables for $\pi^{0}$ production off quasi-free nucleons has also been initiated. Very preliminary results for the target asymmetry $T$ and the double polarization observable $F$ (circularly polarized beam, transversely polarized target) measured at MAMI are shown in Fig. 5. In this case, agreement between free and quasi-free data is better, FSI effects on this polarization observables seem to be smaller than for absolute cross sections.

\subsection{Photoproduction of isoscalar mesons $-\eta$ and $\eta^{\prime}$}

Photoproduction of the "heavy", isoscalar mesons $\eta$ and $\eta^{\prime}$ is interesting because it is selective for $N^{\star}$ resonances. Experimental progress has been substantial over the last few years. Total cross sections and 

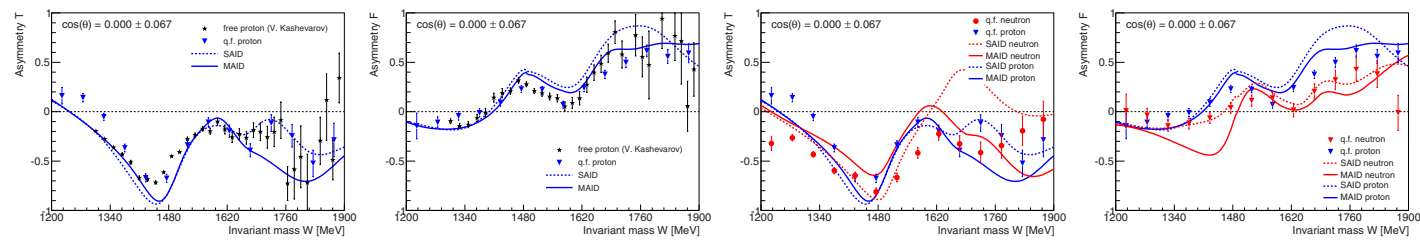

Figure 5. Polarization observables for quasi-free protons and neutrons for the $\gamma N \rightarrow N \pi^{o}$ reaction with transversely polarized target and circularly polarized beam (MAMI). From left to right: (1) target asymmetry free (black stars) and quasi-free (blue triangles) protons, (2) same for observable $F$, (3) $T$ for quasi-free protons (blue triangles) and quasi-free neutrons (red spheres), (4) same for $F$.

angular distributions for $\gamma p \rightarrow p \eta$ and $\gamma p \rightarrow p \eta^{\prime}$ have been studied at ELSA [27-29] and at MAMI [30]. Also for these reactions the isospin degree of freedom has moved into the focus. In contrast to pion production for both mesons it has been found [31-33] that for quasi-free production from nucleons bound in the deuteron FSI effects are negligible. The $\gamma n \rightarrow n \eta$ reaction has attracted interest because an unexpected narrow structure has been observed in the excitation function $[31,35,36]$. Recent measurements at MAMI [33,34] have confirmed this structure with much improved statistical significance not only for neutrons bound in the deuteron but also for the ${ }^{3} \mathrm{He}$ target. It can be phenomenologically fitted with a Breit-Wigner curve with position $W_{R}=(1670 \pm 5) \mathrm{MeV}$, a width of $\Gamma_{R}=(30 \pm 15) \mathrm{MeV}$ and a strength of $\sqrt{b_{\eta}} A_{1 / 2}^{n}=(12.3 \pm 0.8) 10^{-3} \mathrm{GeV}^{-1 / 2}$ [33]. It's nature is not yet understood. The recent measurements have produced precise differential cross sections, which show a non-trivial angular dependence of the structure [33,34]. Currently, first measurements of the helicity dependence of the cross section and the polarization observables $T$ and $F$ are under analysis (MAMI).

\subsection{Photoproduction of pseudoscalar meson pairs}

Photoproduction of meson pairs gives access to excited nucleon states which have no significant decay branching ratios directly to the nucleon ground state but decay via intermediate excited states. The best studied double-meson final state are pion pairs. In the past, total cross sections, invariant mass distributions, and partly also the helicity dependence of the cross section have been measured at ELSA and MAMI for several final states (see e.g. [26, 44] and Ref. therein). However, the complicated amplitude structure of this reaction requires the investigation of further polarization observables. Such a program is currently active at ELSA and MAMI. First results are available for the beamhelicity asymmetry $I^{\odot}(\Phi)=\left(d \sigma^{+}-d \sigma^{-}\right) /\left(d \sigma^{+}+\sigma^{-}\right)$, where $d \sigma^{ \pm}$are the cross sections for the two polarization states of a circularly polarized beam on an unpolarized target and $\Phi$ is the angle between two planes defined by the incident photon and the three final state hadrons (e.g. between the reaction plane defined by photon and recoil nucleon and the production plane defined by the two pions). Results for $\pi^{0} \pi^{0}$ and $\pi^{+} \pi^{0}$ pairs produced off free protons and the corresponding reactions off quasifree protons and neutrons bound in the deuteron have been recently published $[45,46]$. In all cases excellent agreement was found between the results for free and quasi-free protons, indicating that also this observable is basically unaffected by FSI. Agreement with model predictions [43] is not yet good (in particular not for the mixed-charge pairs); such data will certainly better constrain future analyses. Data for further polarization observables (e.g. $T, F$ at MAMI, $G$ at ELSA) are also under analysis.

More recently also the $\eta \pi$ final state attracted much interest. Total cross sections, invariant mass distributions, and also some polarization observables have been measured for $\gamma p \rightarrow p \pi^{0} \eta$ [37-42]. This decay channel is very selective. The $\eta$-meson is isoscalar, so that nucleon resonances can only emit it in $N^{\star} \rightarrow N^{(\star)}$ or $\Delta^{\star} \rightarrow \Delta^{(\star)}$ transitions. The results [38, 41] suggested for the threshold region a dominant contribution from the $D_{33}(1700) \rightarrow \eta P_{33}(1232) \rightarrow \eta \pi N$ cascade. This leads to a simple 

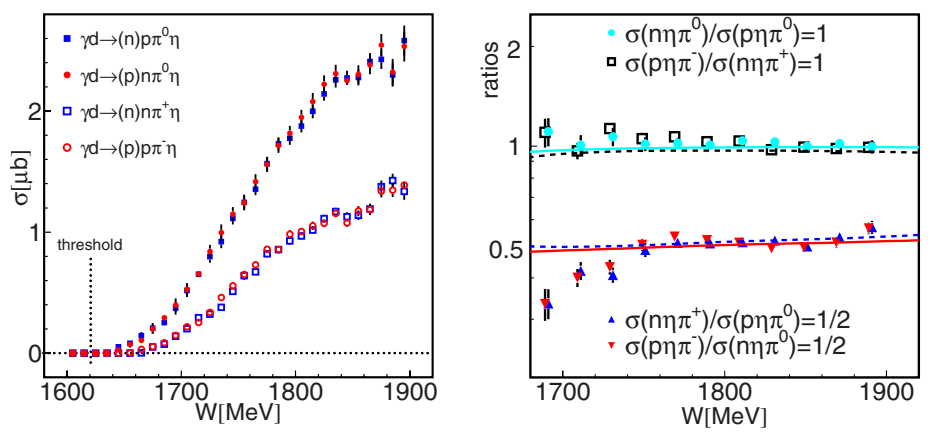

Figure 6. Quasi-free excitation functions for $\gamma N \rightarrow N \eta \pi$ measured with a deuteron target. Left hand side: total cross sections, right hand side: cross section ratios. All data very preliminary. Model curves from [41].

prediction for the cross section ratios of the different charge states: $\sigma\left(\gamma p \rightarrow \eta \pi^{0} p\right)=\sigma\left(\gamma n \rightarrow \eta \pi^{0} n\right)=$ $2 \sigma\left(\gamma p \rightarrow \eta \pi^{+} n\right)=2 \sigma\left(\gamma n \rightarrow \eta \pi^{-} p\right)$, while for the photoexcitation of an $N^{\star}$ resonance the factors 2 would be $1 / 2$ and the cross section ratios for neutron and proton targets could be anything. Preliminary results from MAMI for all four isospin channels are summarized in Fig. 6 and clearly confirm the above relations. The absolute scale of the cross sections for the quasi-free proton bound in the deuteron are suppressed with respect to the free proton by roughly $25 \%-30 \%$, so that significant FSI effects are observed.

\section{Summary}

The experimental programs at ELSA and MAMI aiming at the measurement of cross sections and polarization observables for several single and double meson production reactions off free and quasifree nucleons have very successfully taken data. First results are published and many more results are in preparation. Currently, the experiment at MAMI continues data taking, while the Crystal Barrel experiment at Bonn is in an upgrade phase and will restart data taking in 2015.

The unpublished, preliminary results discussed in this paper are parts of the PhD theses of J. Hartmann (Bonn) and M. Dieterle, A. Käser, and Th. Strub (Basel). This work was supported by Schweizerischer Nationalfonds (200020-132799,121781,117601,113511), Deutsche Forschungsgemeinschaft (SFB 443, SFB/TR 16), DFG-RFBR (Grant No. 05-02-04014), UK Science and Technology Facilities Council, (STFC 57071/1, 50727/1), European Community-Research Infrastructure Activity (FP6), the US DOE, US NSF and NSERC (Canada).

\section{References}

[1] C. Amsler, B. Krusche, T. DeGrand (Quark Model review), in J. Beringer et al., Phys. Rev. D 86, 010001 (2012)

[2] W. Plessas, Mod. Phys. Lett. A 28, 1360022 (2013)

[3] G. Eichmann Prog. Part. Nucl. Phys. 67, 234 (2012)

[4] I.G. Aznauryan et al., Int. J. Mod. Phys. E 22, 1330 (2013)

[5] R.G. Edwards et al., Phys. Rev. D 84, 074508 (2011)

[6] J. Beringer et al., Phys. Rev. D 86, 010001 (2012)

[7] W. Hillert, Eur. Phys. J. A 28, 139 (2006)

[8] K.-H. Kaiser et al., Nucl. Instr. Meth. A 593, 159 (2008)

[9] J.C. McGeorge et al., Eur. Phys. J. A 37, 129 (2008)

[10] D. Elsner et al., Eur. Phys. J. A 39, 373 (2009) 
[11] A. Thomas et al., Phys. of Particles and Nuclei 44, 964 (2013)

[12] A. Starostin et al., Phys. Rev. C 64, 055205 (2001)

[13] E. Aker et al., Nucl. Instr. and Meth. A 321, 69 (1992)

[14] A.R. Gabler et al., Nucl. Instr. and Meth. A 346, 168 (1994)

[15] M. Gottschall et al., Phys. Rev. Lett. 112, 012003 (2014)

[16] R.L. Workman et al., Phys. Rev. C 86, 015202 (2012).

[17] D. Drechsel, S.S. Kamalov, L.Tiator, Eur. Phys. J. A 34, 69 (2007)

[18] A.V. Anisovich et al., Eur. Phys. J. A 44, 203 (2010)

[19] A.V. Anisovich et al., Eur. Phys. J. A 49, 67 (2013)

[20] A. Thiel et al., Phys. Rev. Lett. 109, 102001 (2012)

[21] W. Briscoe et al., GWU analysis center, (2013)

[22] M.H. Sikora et al., Phys. Rev. Lett. 112, 022501 (2014)

[23] W. Chen et al., Phys. Rev. Lett. 103, 012301 (2009)

[24] W. Chen et al., Phys. Rev. C 86, 015206 (2012)

[25] B. Krusche, Eur. Phys. J. Special Topics 198, 199 (2011)

[26] B. Krusche and S. Schadmand, Prog. Part. Nucl. Phys. 51, 399 (2003)

[27] V. Crede et al., Phys. Rev. Lett. 94, 012004 (2005)

[28] O. Bartholomy et al., Eur. Phys. J. A 33, 133 (2007)

[29] V. Crede et al., Phys. Rev. C 80, 055202 (2009)

[30] E.F. McNicoll et al., Phys. Rev. C 82, 035208 (2010)

[31] I. Jaegle et al., Eur. Phys. J. A 47, 89 (2011)

[32] I. Jaegle et al., Eur. Phys. J. A 47, 11 (2011)

[33] D. Werthmüller et al., Phys. Rev. Lett. A 49, 154 (2013)

[34] L. Witthauer et al., Eur. Phys. J. 111, 232001 (2013)

[35] V. Kuznetsov et al., Phys. Lett. B 647, 23 (2007)

[36] I. Jaegle et al., Phys. Rev. Lett. 100, 252002 (2008)

[37] I. Horn et al., Phys. Rev. Lett. 101, 202002 (2008)

[38] I. Horn et al., Eur. Phys. J. A 38, 173 (2008)

[39] E. Gutz et al., Eur. Phys. J. A 35, 291 (2008)

[40] E. Gutz et al., Phys. Lett. B 687, 11 (2010)

[41] V. Kashevarov et al., Eur. Phys. J. A 42, 141 (2009)

[42] V. Kashevarov et al., Phys. Lett. B 693, 551 (2010)

[43] A. Fix and H. Ahrenhövel, Eur. Phys. J. A 25, 115 (2005)

[44] A.V. Sarantsev et al., Phys. Lett. B 659, 94 (2008)

[45] M. Oberle et al., Phys. Lett. B 721, 237 (2013)

[46] M. Oberle et al., Eur. Phys. J. A (2014), in press 